Conclusions: This survey suggested that CTD have a major impact on FP and family size, possibly mediated by the increased rate of miscarriages as compared to CA. Concerns about reproductive issues could be positively overcome by adequate counselling.

Rheumatologists should implement the discussion about FP and the compatibility of drugs with pregnancy in the management of young women with RD, especially those with CTD for whom contraception and pregnancy have particular implications.

Acknowledgements: Statistical analysis supported by an unrestricted grant by UCB Pharma

Thanks to Patients' Associations and Participants

Disclosure of Interest: None declared

DOI: 10.1136/annrheumdis-2017-eular.2268

\section{AB1100 PATIENT SELF-MANAGEMENT APPS AS ONE MODULE OF AN INTEGRATED TIGHT-CONTROL CONCEPT BASED ON THE EXAMPLES OF THE DIGITAL APPLICATIONS RHEUMALIVE UND AXSPALIVE}

M. Feuchtenberger ${ }^{1}$, S. Kleinert ${ }^{2}$, F. Schuch ${ }^{2}$, S. Spähtling-Mestekemper ${ }^{3}$ C. Kuhn ${ }^{4}$, M. Welcker ${ }^{5} .{ }^{1}$ Rheumatologie, Schwerpunktpraxis für Rheumatologie, Burghausen; ${ }^{2}$ Rheumatologie, Rheumatologische Schwerpunktpraxis, Erlangen; ${ }^{3}$ Rheumatologie, Rheumapraxis München-Pasing, München; ${ }^{4}$ Rheumatologie, internistische Schwerpunktpraxis am Ludwigsplatz, Karlsruhe; ${ }^{5}$ Rheumatologie, MVZ für Rheumatologie Dr. Martin Welcker, Planegg, Germany

Background: Medical Applications have the potential to support physicians and patients to document the course of the disease and optimize therapies. We describe the linkage and integration of patient reported outcomes (PROs) into existing medical office software and rheumatologic documentation systems by means of the digital applications RheumaLive and AxSpALive. RheumaLive is certified as medical product class I, and certification for AxSpALive is in preparation.

Methods: RheumaLive and AxSpALive are applications that can be downloaded free of charge from the common app-stores (Android, Apple) and installed on mobile devices (smartphones, tablets). A freeware version for PCs is also available (Windows- und Mac OS X-Version). A diary functionality allows to document the medication uptake (partially filed, memory-functionality for medication uptake available), validated scores like VAS, SJC, morning stiffness, FFBh (comparable to HAQ), BASDAI, RADAl, etc. and days of sick leave (+/- related to the rheumatic disease). All App-versions are password-protected, which is provided by the treating rheumatologist (relationship patient-physician).

The patient reported outcomes are stored locally on the respective device. For the subsequent visit, they can be printed or sent encrypted to the treating physician via internet in a prespecified interval. A specific software allows to integrate the data into the existing medical office software.

In daily practise, data entered by patients into RheumaLive can be screened and compared with collected clinical data and laboratory measures, because of it's certification as medical product. In case of an increasing disease activity the patient can be rapidly contacted and medication can be modified, if necessary. Regular F2F-appointments remain integral part of the medical care, even in case of good controlled disease activity. But in case of F2F-appointments, PROs can be delivered by patients, saving time and resources.

The PROs can be made available to rheumatologic documentation systems like RheumaDok via predefined interfaces.

Results: In a first pilot phase 54 patients in 4 rheumatologic specialised practices were documented. In the next phase the number of patients and participating practices/centres shall be increased.

Further studies are necessary to show if the integration of PROs which are collected via electronical application can lead to an improvement of rheumatologic diseases.

Conclusions: The integration of PROs collected with RheumaLive and AxSpALive into existing rheumalogic documentation systems allows a close monitoring of disease courses and therapies according to the "tight control" principle.

Acknowledgements: RheumaLive and AxSpALive werde developed by Starhealth $\mathrm{GmbH}$ on behalf of UCB Pharma GmbH.

Disclosure of Interest: M. Feuchtenberger Speakers bureau: UCB, S. Kleinert Speakers bureau: UCB, F. Schuch Speakers bureau: UCB, S. SpähtlingMestekemper Speakers bureau: UCB, C. Kuhn: None declared, M. Welcker Speakers bureau: UCB

DOI: 10.1136/annrheumdis-2017-eular.5782

\section{AB1101 QUALITY OF LIFE AND WORK IN PATIENTS WITH ANKYLOSING SPONDYLITIS OF WORKING AGE}

M. Parvu ${ }^{1}$, G.M. Muresan ${ }^{2}$, A.I. Cozos ${ }^{3}$, I.A. Biro ${ }^{1}$, H.V. Popoviciu ${ }^{1}$, S. Szasz ${ }^{1}$, S. Voidazan ${ }^{4} .{ }^{1}$ Rheumatology, Rehabilitation, University of Medicine and Pharmacy Tg.Mures, Emergency County Hospital; ${ }^{2}$ Internal Medicine; ${ }^{3}$ Rheumatology, Rehabilitation, Emergency County Hospital; ${ }^{4}$ Epidemiology, University of Medicine and Pharmacy, Tg.Mures, Romania

Background: The assessment of the quality of life is a current research issue in patients with ankylosis spondylitis ${ }^{1,2}$. The tools for assessing the life quality, come to complete the other methods of investigation, which assess in a subjective manner the negative impact of the disease on daily activities.

Objectives: The purpose of this study is to define the functional ability, the disease activity, to investigate the correlation between work and quality of life, and the impact of the quality of life in patients with AS.

Methods: The study is an observational prospective study, which includes a total of 91 patients defined with AS in according to the modified New York criteria, from the Rheumatology Clinic of Targu-Mures. Data were obtained by questionnaires including several generic and disease related Quality of life tools. It includes specific tools: BASDAI (Bath Ankylosing Spondylitis Disease Activity Index); BASFI (Bath Ankylosing Spondylitis Functional Index); ASQOL (Ankylosing Spondylitis Quality of Life); and generic tools:SF-36 (Short-Form 36), Eq 5D (European-Quality of life 5 Dimensions), HAQ (Health Assessment Questionnaire and VAS (Visual Analogue Scale).

Results: Out of 91 patients, $82 \%$ were males, $18 \%$ were females; mean age (years) 50,27 $\pm 11,05 \mathrm{SD}$; age at disease onset (years)36,20 $\pm 13,51 \mathrm{SD}$; duration

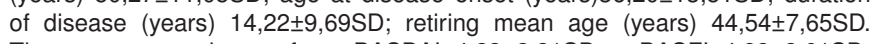
The mean values for $\mathrm{BASDAl}=4,23 \pm 2,21 \mathrm{SD}$; $\mathrm{BASF}=4,88 \pm 2,61 \mathrm{SD}$; $E Q-5 D=5,71 \pm 0,20 S D ; \quad E Q-V A S=64,26 \pm 18,938 D ; \quad H A Q=0,747 \pm 0,51 S D ; \quad S F-$ physical summary $=33,53 \pm 8,33 \mathrm{SD}$; SF-emotional summary $=47,57 \pm 10,03 \mathrm{SD}$; ASQOL $=6,94 \pm 2,68 \mathrm{SD}$. We found a positive correlation between the disease onset and retiring age $p<0,0001, r=0,731 a$ nd a negative significant correlation between BASFI and SFphysical summary $(p<0,0001, r=-0,798)$, ASQOL and SF-emotional summary, $p<0,0001, r=-0,595)$.

Conclusions: The results showed the invalidant potential of the AS, with major impact upon the quality of life.

\section{References:}

[1] Van der Heijde D, Landewe R. Assessment of disease activity, function and quality of life. In 1st edition Philadelfia:Mosby Elsevier;2006, 206-213.

[2] Dovard LC, McKenna SC, Meads MD et all. Translation and Validation of non English version of the ASQOL questionnaire. BioMed Central, Health and QOL outcomes, 2007, 5L7 doiL1.1186/1477-7525-5-7.

Disclosure of Interest: None declared

DOI: 10.1136/annrheumdis-2017-eular.6559

\section{AB1102 LOW BONE MINERAL DENSITY IS A MAJOR CONTRIBUTOR IN THE EUROPEAN HEALTH BURDEN DUE TO ROAD TRAFFIC ACCIDENTS IN PEOPLE AGED 50 YEARS AND ABOVE}

N. Wilson ${ }^{1}$, L. Sanchez-Riera ${ }^{2}$, D. Prieto-Alhambra ${ }^{3}$, C. Cooper ${ }^{4}$,

K. Dreinhöfer ${ }^{5}$, A. Woolf ${ }^{6}$, L. March ${ }^{1}$, P. Halbout ${ }^{7} .{ }^{1}$ Institute of Bone and Joint Research, University of Sydney, Sydney, Australia; ${ }^{2}$ University Hospital Bristol NHS Foundation Trust, Bristol; ${ }^{3}$ Oxford NIHR Musculoskeletal Biomedical Research Unit, University of Oxford, Oxford; ${ }^{4}$ MRC Lifecourse Epidemiology Unit, University of Southampton, Southampton, United Kingdom; ${ }^{5}$ Center for Musculoskeletal Surgery, Charité Universitätsmedizin, Berlin, Germany;

${ }^{6}$ Institute of Health Research, University of Exeter Medical School, Exeter, United Kingdom; ${ }^{7}$ International Osteoporosis Foundation, Nyon, Switzerland

Background: Road traffic accidents (RTAs) are the second leading injury health burden and cause of death in Europe, after falls (1). A significant but as yet unreported proportion of such burden is potentially due to low bone mineral density (BMD), especially among older people, through its relationship with fractures.

Objectives: To measure the percentage of deaths, disability-adjusted life years (DALYs) and years lived with disability (YLDs) due to RTA in people aged 50 years and above attributable to low BMD in the European population for the year 2015. Methods: The estimates followed the Counterfactual Risk Assessment Methodology used in the GBD study (1). Systematic review was performed seeking population-based studies with femoral neck BMD (FNBMD) measured by Dual-XRay-Absorptiometry in people 50 years and over. Age- and sex-specific levels of mean +/-SD FNBMD $\left(\mathrm{g} / \mathrm{cm}^{2}\right)$ were extracted from eligible studies, and this was used as the exposure variable. The age and sex-specific 99 th percentile from nonHispanic whites in National Health and Nutrition Examination Survey (NHANES) 2009-2010 was used as theoretical minimum risk factor exposure distribution, to estimate the potential impact fraction (PIF) of FNBMD for fractures. Relative risks of FNBMD for fractures were obtained from a previous meta-analysis (2). Attributable deaths due to RTA-related fractures were obtained through coded hospital data. Disability levels were established by applying disability weights to each type of fracture. Then, PIFs were applied to obtain attributable deaths and disability due to low BMD.

Results: In the European population aged $50-69$ and 70 years and above, $10.8 \%(95 \% \mathrm{Cl}: 8.9-12.4 \%)$ and $30.9 \%(29.1-32.4 \%)$ of RTA-related deaths, respectively, were attributable to low BMD. In the age group 50-69 this was the second most important risk factor following alcohol use and in those 70 years and above became the most important risk factor, with double the weight of alcohol use. This represents 2,537 and 5,460 absolute deaths in those aged 50-69 and 70 years and above, respectively. The percentage of health burden and disability caused by RTAs attributable to low BMD grew steadily from the ages of 50 and onwards.

Conclusions: This data shows the non-previously reported important role of low $\mathrm{BMD}$ as a preventable risk factor for European RTAs' health burden in population 50 years and over, which requires urgent attention. 


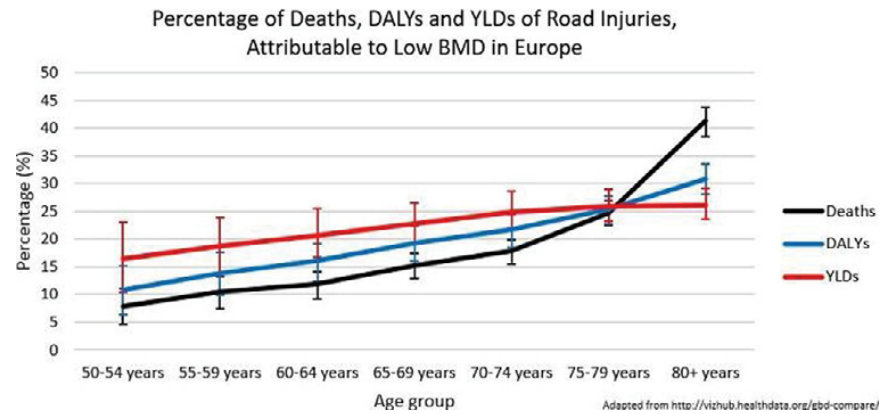

References:

[1] Forouzanfar et al. Lancet 2016.

[2] Johnell et al. JBMR 2005.

Disclosure of Interest: None declared

DOI: 10.1136/annrheumdis-2017-eular.3278

\section{AB1103 THE IMPORTANCE OF ADEQUATE SCREENING TO AVOID FALSE POSITIVES IN THE DIAGNOSIS OF RHEUMATOID ARTHRITIS}

P. Santos-Moreno ${ }^{1}$, D. Gomez ${ }^{1}$, E. Castillo ${ }^{1}$, R. Giraldo ${ }^{1}$, G. Ballesteros ${ }^{1}$, L. Villarreal ${ }^{2}$, D. Buitrago-Garcia ${ }^{3}$, J. Bello ${ }^{3} \cdot{ }^{1}$ Rheumatology; ${ }^{2}$ Psychology and processes; ${ }^{3}$ Epidemiology, Biomab, Center for Rheumatoid Arthritis, Bogota, Bogota, Colombia

Background: The lack of expertise and skills in the diagnosis of in rheumatoid arthritis (RA) in primary level of Colombian medical centers can cause misdiagnosis of rheumatic diseases. Due to this issue in a specialized center in RA we stablished a multidisciplinary model and a strict disease management algorithm to diagnose properly our patients; as a consequence we have achieved the accurate diagnosis of great proportion of patients that were false positives diagnosed initially as RA.

Objectives: The aim of this study was to show effectiveness and accuracy of a screening method to avoid false-positive diagnosis of RA in a cohort of patients with supposed diagnosis of RA.

Methods: During two years we evaluated patients with presumptive diagnosis of RA. We conducted a cross-sectional study; we included patients who were referred from primary care centers to a RA specialized center in a 24 month period with presumptive diagnosis of (RA). Each patient was evaluated to confirm or rule-out diagnosis of RA as follows: a rheumatologist fulfilled a complete medical record, including joint counts; it was assessed rheumatoid factor and anti-citrullinated antibodies, and other laboratories depending on each case. Also were made x-rays of hands and feet, and in some cases of persistent doubt about the diagnosis was requested comparative MRI of hands or/and feet. Descriptive epidemiology was perfomed.

Results: Between 2015 and 20166813 patients were evaluated in our specialized center, in $76 \%$ of cases RA was confirmed, the remaining 1593 patients (24\%) had a wrong diagnosis of RA; of these misdiagnosed patients, $(87 \%)$ were female, and $205(13 \%)$ male, with an average age of $62 \pm 12$ years. Between differential diagnosis which were found in this cohort of misdiagnosed patients: osteoarthritis in 849 patients (63.3\%), Sjögren syndrome (7\%) Systemic lupus erythematosus (6\%) the remaining $30 \%$ of patients had conditions such as gout, psoriasis, osteoporosis, myalgia, soft tissue diseases among others. The majority of patients with wrong diagnosis took DMARDs $(23 \%)$, calcium (11\%), biologics $(10 \%)$ acetaminophen $(9 \%)$, neuropathy medications $(7 \%)$, acetaminophen plus opioids $(5 \%)$, osteoporosis medications $(5 \%)$, opioids $(4 \%)$, glucosamine $(4 \%)$, diacerein $(3 \%)$, the remaining patients took medications such as NSAID, glucosamine, antigout agents, gastritis drugs, among others.

Conclusions: The results of this program show that almost $25 \%$ patients with presumptive RA diagnosis are misdiagnosed; this is evidence that can be extrapolated to primary care centers in Colombia. The most important cofounding diagnosis was osteoarthritis and many patients were receiving DMARDs for treatment. For this reason there is an urgent need of education strategies for primary care physicians and the implementation of centers of excellence in RA, in order to conduct a proper diagnose and avoid clinical and health economics consequences of misdiagnosis.

Disclosure of Interest: None declared

DOI: 10.1136/annrheumdis-2017-eular.5249

\section{AB1104 COST-EFFECTIVENES ANALYSIS OF TNF INHIBITORS USE COMPARED TO DMARDS IN THE FATAL AND NONFATAL ACUTE CORONARY ISCHEMIC EVENT}

R.K.S. Gomes ${ }^{1}$, J.Y.K. Viscondi ${ }^{2}$, M.R.C. Nobre ${ }^{2}{ }^{1}{ }^{1}$ Center of specialty of the municipality of Blumenau and Brusque, Blumenau; ${ }^{2}$ São Paulo University, São Paulo, Brazil

Background: Epidemiological studies have established that rheumatoid arthritis is associated with an increase in cardiovascular disease ${ }^{1,2}$. The evaluation of tumour necrosis factor inhibitors (TNFi) on the reduction of the risk of acute myocardial infarction and death due to cardiovascular causes has shown promising results ${ }^{3}$. The economic evaluation for these outcomes are not established yet.

Objectives: To evaluate the cost-effectiveness of TNFi versus disease-modifying antirheumatic drugs (Dmards) to avoid a new case of acute ischemic heart disease and death in rheumatoid arthritis patients.

Methods: A cost-effectivenes analysis (CEA) was performed using a Markov model 6-month transition cycle, with time horizon of 30 years, under the Brazilian public healthcare system perspective. Costs are expressed in 2015 Reais and effectiveness measures are new cases of acute ischemic coronary disease and cardiovascular death.

Results: The average cost in 30 years of Dmards and TNFi was 14,291,105.28 and $96,151,873.86$ Reais, respectively. The incremental effectiveness was 2.69 cases of coronary artery disease and consequent incremental cost-effectiveness ratio (ICER) of 30,527,502.27 Reais per new cases avoided, while for cardiovascular death, incremental effectiveness was 1.33 and an ICER of 61,634,231,69 Reais per new cases avoided. The univariate analysis identified that the most relevant parameter in the ICER on both outcomes was the TNFi drug. The sensitivity analysis established that, in order to, reach the amount of willingness to pay (WTP) per semester to avoid an acute myocardial infarction, the average cost of TNFi should be 1,337.47 Reais per case avoided and the average cost for the cardiovascular death avoidence sould be 954.22 Reais. All the analyzes performed establish an unfavorable relationship of the drug treatment strategy with TNFi.

Conclusions: The findings of the CEA among patients with rheumatoid arthritis for cardiovascular outcomes when compared to the strategy of TNFi drug treatment with the dominant strategy Dmards after the first 6 months of exposure point out an unfavorable relationship, surpassing the amount of expenses recommended by the Ministry of Health of Brazil in the year 2015.

\section{References:}

[1] Symmons DP, Gabriel SE. Epidemiology of CVD in rheumatic disease, with a focus on RA and SLE. Nat Rev Rheumatol. 2011;7(7):399-408.

[2] Bergström U, Jacobsson LT, Turesson C. Cardiovascular morbidity and mortality remain similar in two cohorts of patients with long-standing rheumatoid arthritis seen in 1978 and 1995 in Malmö, Sweden. Rheumatology (Oxford). 2009;48(12):1600-5.

[3] Dixon WG, Watson KD, Lunt M, Hyrich KL; British Society for Rheumatology Biologics Register Control Centre Consortium. Reduction in the incidence of myocardial infarction in patients with rheumatoid arthritis who respond to anti-tumor necrosis factor alpha therapy: results from the British Society for Rheumatology Biologics Register. Arthritis Rheum. 2007;56(9):2905-12.

Acknowledgements: Foundation for Research Support of the State of São Paulo, FAPESP 2013/12979-1.

Disclosure of Interest: None declared

DOI: 10.1136/annrheumdis-2017-eular.2124

\section{AB1105 FACTORS IMPACTING PATIENTS' DECISION TO START TREATMENT VARY BY RACE}

R. Cozmuta ${ }^{1}$, L. Fraenkel ${ }^{2} .{ }^{1}$ Rheumatology, Emory University, Atlanta; ${ }^{2}$ Yale University, New Haven, United States

Background: Previous research has found that young minority women tend to be more risk averse compared to their Caucasian counterparts. The reasons underlying these differences, however, are not understood.

Objectives: The objective of this study was to examine whether factors influencing perceived treatment importance vary by race.

Methods: Women between the ages of 20 and 45 completed a survey. The survey recorded sociodemographic data, trust in healthcare systems and beliefs in medications. It also included a hypothetical scenario in which subjects were asked to rate the importance of taking a medication for a patient with joint pain, migraines and fatigue that benefits $70 \%$ of people and is well tolerated except for the rare risk (1 per 100,000) of a neurologic disease that may cause weakness, trouble with vision and numbness. Associations between patient characteristics, medication beliefs, and trust with perceived importance of taking the medication were evaluated for each race. Variables found to be statistically significant were subsequently evaluated using multiple linear regression.

Results: 299 women completed the survey. Baseline characteristics by ethnicity are described in Table 1. Hispanic women had more negative medication beliefs than did Non-Hispanic Whites, and both Non-Hispanic Black and Hispanic

Table 1. Patient characteristics by race

\begin{tabular}{lcccc}
\hline Variable & Non-Hispanic White & Non-Hispanic Black & Hispanic & P value \\
\hline Age (mean, SD) & $34.1(7.2)$ & $35.2(8.4)$ & $32.8(8.6)$ & 0.1 \\
Poor self-reported health (\%) & 12 & 14 & 12 & 0.9 \\
Some college education (\%) & 75 & 61 & 43 & $<0.01$ \\
Income <12,000/year (\%) & 23 & 33 & 34 & 0.2 \\
Difficulty paying for meds (\%) & 68 & 58 & 60 & 0.3 \\
Medication beliefs (mean, SD) & $20.6(4.6)$ & $21.6(4.4)$ & $22.3(4.7)$ & 0.04 \\
Trust (mean, SD) & $27.9(5.5)$ & $28.6(5.7)$ & $29.1(5.5)$ & 0.3 \\
Hopeful (mean, SD) & $4.2(1.6)$ & $3.5(1.7)$ & $3.6(1.7)$ & $<0.01$ \\
Worried (mean, SD) & $4.6(1.6)$ & $4.8(1.8)$ & $5.1(1.6)$ & 0.2 \\
Important (mean, SD) & 5.3 & 5.6 & 5.5 & 0.3 \\
\hline
\end{tabular}

